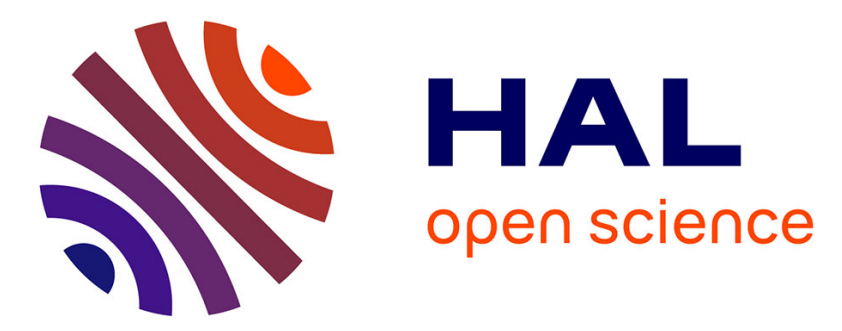

\title{
Atom probe informed simulations of dislocation-precipitate interactions reveal the importance of local interface curvature
}

A. Prakash, Julien Guénolé, J. Wang, J. Müller, E. Spiecker, M.J. Mills, I. Povstugar, P. Choi, D. Raabe, E. Bitzek

\section{To cite this version:}

A. Prakash, Julien Guénolé, J. Wang, J. Müller, E. Spiecker, et al.. Atom probe informed simulations of dislocation-precipitate interactions reveal the importance of local interface curvature. Acta Materialia, 2015, 92, pp.33-45. 10.1016/j.actamat.2015.03.050 . hal-02459286

\section{HAL Id: hal-02459286 \\ https://hal.science/hal-02459286}

Submitted on 7 May 2020

HAL is a multi-disciplinary open access archive for the deposit and dissemination of scientific research documents, whether they are published or not. The documents may come from teaching and research institutions in France or abroad, or from public or private research centers.
L'archive ouverte pluridisciplinaire HAL, est destinée au dépôt et à la diffusion de documents scientifiques de niveau recherche, publiés ou non, émanant des établissements d'enseignement et de recherche français ou étrangers, des laboratoires publics ou privés. 


\title{
Atom probe informed simulations of dislocation-precipitate interactions reveal the importance of local interface curvature
}

\author{
A. Prakash ${ }^{\mathrm{a}}$, J. Guénoléa, J. Wang ${ }^{\mathrm{a}}$, J. Müller ${ }^{\mathrm{b}}$, E. Spiecker ${ }^{\text {b }}$, M. J. Mills ${ }^{\mathrm{c}}$, I. Povstugar ${ }^{\mathrm{d}}$, P. Choi $^{\mathrm{d}}$, \\ D. Raabe ${ }^{\mathrm{d}}$, E. Bitzek $^{\mathrm{a}, *}$ \\ ${ }^{a}$ Department of Materials Science and Engineering, Institute I, \\ Friedrich-Alexander-Universität Erlangen-Nürnberg (FAU), 91058 Erlangen, Germany \\ ${ }^{b}$ Department of Materials Science and Engineering, Institute IX, \\ Friedrich-Alexander-Universität Erlangen-Nürnberg (FAU), 91058 Erlangen, Germany \\ ${ }^{c}$ Department of Materials Science and Engineering, The Ohio State University, Columbus, OH 43210, USA \\ ${ }^{d}$ Department of Microstructure Physics and Alloy Design, \\ Max-Planck-Institut für Eisenforschung, 40237 Düsseldorf, Germany
}

\begin{abstract}
The interaction of dislocations with precipitates is an essential strengthening mechanism in metals, as exemplified by the superior high-temperature strength of Ni-base superalloys. Here we use atomistic simulation samples generated from atom probe tomography data of a single crystal superalloy to study the interactions of matrix dislocations with a $\gamma^{\prime}$ precipitate in molecular dynamics simulations. It is shown that the precipitate morphology, in particular its local curvature, and the local chemical composition significantly alter both, the misfit dislocation network which forms at the precipitate interface, and the core structure of the misfit dislocations. Simulated tensile tests reveal the atomic scale details of many experimentally observed dislocation-precipitate interaction mechanisms, which cannot be reproduced by idealized simulation setups with planar interfaces. We thus demonstrate the need to include interface curvature in the study of semicoherent precipitates and introduce as an enabling method atom probe tomography-informed atomistic simulations.
\end{abstract}

Keywords: Ni-base superalloys; Precipitation hardening; Misfit dislocation network; Atomistic simulation; Atom probe tomography (APT)

\section{Introduction}

Ni-base superalloys are key materials for single crystal turbine blades in gas turbines of aero-engines and power plants $[1,2]$. Their ability to withstand mechanical loads at high temperatures is mostly due to their $\gamma / \gamma^{\prime}$ microstructure, consisting of about 70 vol.\% cuboidal precipitates of the ordered $\mathrm{L}_{2} \gamma^{\prime}$ phase embedded in a face-centred cuboidal (fcc) solid solution matrix. The increased strength of these two-phase superalloys, compared to the individual phases, is a direct result of the coherent $\gamma / \gamma^{\prime}$ interfaces [3]. During the initial stages of high temperature, low stress creep, the lattice misfit between the two phases is relieved by the deposition of dislocation segments at the $\{100\}-\gamma / \gamma^{\prime}$ interfaces by dislocations gliding in the $\gamma$-channels between the precipitates $[4,5,6]$. These dislocation segments react with each other and locally rearrange to form an interfacial misfit dislocation network $[7,8,9,10]$. This process is usually accompanied by a change of the precipitate shape from cuboidal to a lamellar structure (rafting) [11, 12]. This rafted microstructure determines the creep of single crystalline superalloys by effectively hindering the annihilation of dislocations of opposite sign by climb processes $[13,14]$. The interfacial dislocation network plays a key role in protecting the $\gamma^{\prime}$ precipitate by preventing dislocations from the $\gamma$ channels to cut into the $\gamma^{\prime}$ phase $[15,10]$.

\footnotetext{
* Corresponding author

Email address: erik.bitzek@fau.de (E. Bitzek)
} 
The present knowledge of dislocation-precipitate interactions is based almost exclusively on post-mortem transmission electron microscopy (TEM) studies of dislocation "relics" from which the underling formation processes can only be inferred. These critical dynamic interaction processes can on the other hand be directly explored through large-scale atomistic simulations, which can capture the interaction mechanisms at the dislocation core level. However, only very few such simulations have been reported in the literature [16, 17, $18,19,20,21,22]$, and almost all of them make use of highly idealized simulation setups with perfectly planar inter-phase boundaries (IPB) and periodic boundary conditions (PBC) [17, 18, 19, 20, 21, 22]. In particular, the interaction of matrix dislocations with the interfacial dislocation network only recently became the focus of an atomistic study: Zhu et al. [22] studied the interaction of infinite, straight screw dislocations with a misfit dislocation network on a planar IPB in a quasi-2D setup at $0 \mathrm{~K}$.

The use of highly idealized, quasi-2D simulation scenarios is typical for atomistic simulations. While simplified, highly controlled setups are often necessary to quantitatively determine material properties, overly simplified setups might artificially suppress important mechanisms. Examples include the use of PBC along the crack front in nearly all studies on fracture which suppresses kink formation and crack front curvature effects [23, 24], neglecting surface roughness in the study of nanowires where it was shown to significantly influence dislocation nucleation and the overall deformation behaviour [25], and the nearly ubiquitous use of Voronoi tessellation in simulations of nanocrystalline metals resulting in perfectly planar grain boundaries (GBs) which fail to represent the GB network topology of real materials [26].

Ideally, one would like to be able to perform simulations on samples which are atom-by-atom reproductions of the experimental specimens. Atom probe tomography (APT), which combines time of flight spectroscopy on individual ions that are sequentially evaporated from a sharp tip with a position-sensitive detector [27, 28] (Fig. 1a), could provide exactly this kind of 3D information on the position and chemical species of atoms within a needle-shaped specimen. APT measurements are therefore increasingly used in conjunction with atomisitic simulations. APT data were used to construct representative idealized models, e.g. for (kinetic) Monte Carlo [29, 30] and Molecular Dynamics (MD) [31] simulations, or density functional theory (DFT) calculations $[32,33,34,35]$. The simulation results were subsequently either compared to experiments or used to evaluate the experimental findings. Only rarely, APT data have been directly used for constructing the actual atomistic simulation samples [35]. This is due to the partial loss of evaporated ions (detection efficiency is $37-80 \%$ depending on the equipment) and field evaporation artefacts affecting the accuracy of reconstructed atomic positions. Recently, new approaches were proposed to create complete atomistic samples based on APT data [35, 36, 37]. Here, a key element is the reconstruction of the lattice configuration of the original sample (lattice rectification), which requires real-space spatial distribution maps [37] or the use of Fourier transformations [36]. The lattice rectification replaces the missing atoms, but without reproducing the correct short-range order (SRO). Statistical analysis of the original APT data in combination with a Monte Carlo algorithm to interchange atoms can then be used to match the real SRO $[35,38]$.

In the present work, we suggest a simpler alternative approach to generate APT-informed atomistic simulation samples and use it to obtain a real $\gamma / \gamma^{\prime}$ microstructure directly from a reconstructed APT sample of the alloy ERBO/1 [39]. By comparing the simulation results using this microstructure with the results of simulations on typical idealized simulation setups with planar IPBs, we show that the misfit dislocation network, and in particular the core structure of the misfit dislocations, depend on the precipitate morphology and play a crucial role in determining the possible interaction mechanisms between matrix dislocations and the misfit dislocation network.

\section{Methods}

\subsection{Experiments}

The key idea of the present study lies in conducting APT-informed atomistic simulations. For this purpose, as a first step APT characterization was conducted on a single crystal Ni-base superalloy ERBO/1 with a composition close to the commercial CMSX-4 alloy [39]. In a second step atomistic starting configurations for subsequent MD simulations were generated from the APT data set as outlined in the ensuing 


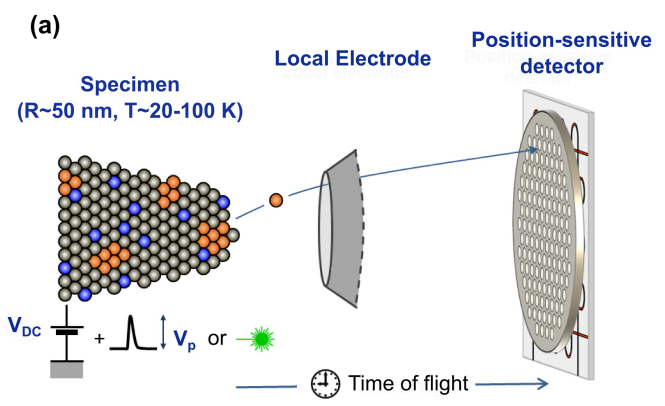

(e)

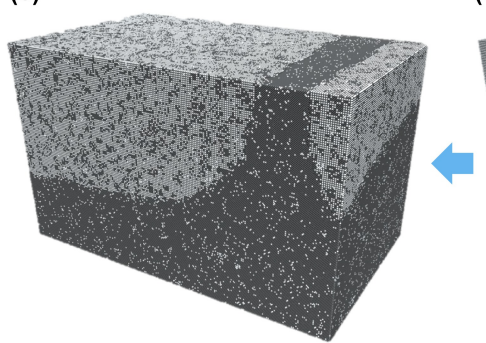

(d)

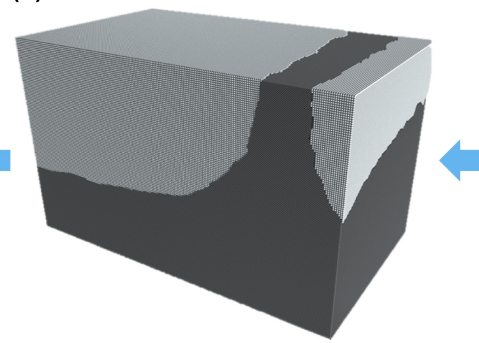

(b)

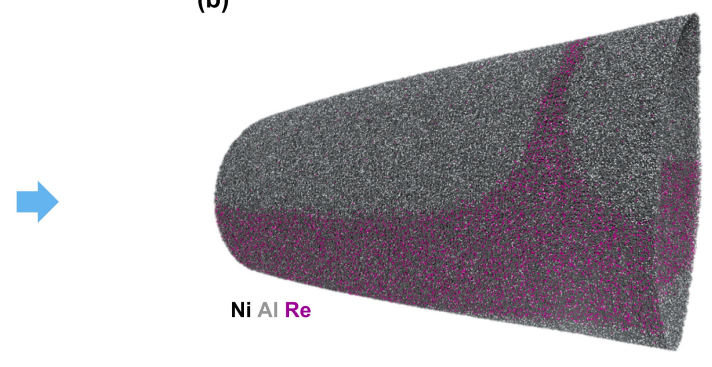

(c)

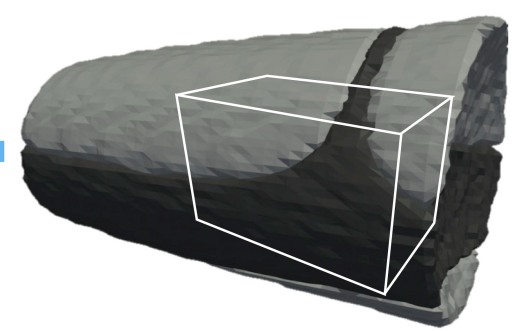

Figure 1: Schematics of the APT setup and of the generation of atomistic simulation samples from APT data. (a) Illustration of the working principle of APT. (b) Reconstructed APT specimen showing only Ni (black), Al (grey), and Re (red) ions. (c) Iso-density surfaces of $\mathrm{Al}$ (grey) and Re (black) determined from the reconstructed APT specimen. The superposed white lines indicate the desired cuboidal box for atomistic simulations. (d) APT-informed sample with stoichiometric chemical composition (pure $\mathrm{Ni} / \mathrm{Ni}_{3} \mathrm{Al}$ ). (e) Non-stoichiometric APT-informed atomistic sample obtained by stochastically replacing atoms in the configuration shown in (d) so as to match the local distributions of Ni and Al in the APT-specimen.

section. The master melt was provided by Cannon-Muskegon and cast into a single crystal plate with $\langle 001\rangle$ orientation along the solidification direction by Doncasters Precision Casting, Bochum. The cast plate was homogenized at $1300^{\circ} \mathrm{C}$ for $6 \mathrm{~h}$ to minimize elemental segregation at the dendrite scale. The homogenized plate was then aged in two steps, $1140^{\circ} \mathrm{C}$ for $4 \mathrm{~h}$ and subsequently at $870^{\circ} \mathrm{C}$ for $16 \mathrm{~h}$, to form a common $\gamma / \gamma^{\prime}$ superalloy microstructure. The alloy was cooled in air after each step of heat treatment process. The actual chemical composition of the alloy after heat treatment was determined by inductive-coupled plasma atomic emission spectroscopy (ICP-AES) and is presented in Table 1.

Specimens for APT analyses were prepared with $\langle 001\rangle$ crystallographic orientation parallel to specimen axis using a dual-beam focused-ion-beam (FIB) system (FEI Helios Nanolab 600). A conventional lift-out method described in [40] was applied. To eliminate the subvolume damaged by Ga ions during preparation, final shaping of the APT tips was performed using low-energy (5 keV) ions. APT measurements were performed using a reflectron-equipped local electrode atom probe (LEAP 3000X HR, Cameca Instruments) in pulsed voltage mode with pulse rate of $200 \mathrm{kHz}$ and pulse fraction of $15 \%$. The specimen base temperature of $60 \mathrm{~K}$ and detection rate of 0.005 ions per pulse were maintained throughout the analysis. Reconstruction, visualization and analysis of APT data sets were performed using the commercial software IVAS 3.6.6 (Cameca Instruments). From the initial APT data set obtained from the experiment about 1 million ions were discarded to eliminate the outer Ga contaminated regions.

Table 1: Chemical composition of the alloy ERBO/1 as measured by ICP-AES.

\begin{tabular}{|l|rrrrrrrrrr|}
\hline Element & $\mathrm{Al}$ & $\mathrm{Co}$ & $\mathrm{Cr}$ & $\mathrm{Hf}$ & $\mathrm{Mo}$ & $\mathrm{Re}$ & $\mathrm{Ta}$ & $\mathrm{Ti}$ & $\mathrm{W}$ & $\mathrm{Ni}$ \\
\hline wt.\% & 5.6 & 9.6 & 6.5 & 0.1 & 0.6 & 2.9 & 6.1 & 1.0 & 6.6 & Bal. \\
\hline
\end{tabular}




\subsection{Atomistic sample generation from APT data}

To construct APT-informed atomistic simulation samples we suggest here a simpler alternative to the approaches currently found in the literature $[36,35,37]$. The fundamental step is to obtain information on the IPB from the APT data. The $\gamma$ and $\gamma^{\prime}$ phases have different chemical compositions, and hence the IPB position can be defined by the iso-density surfaces of any ion which partitions strongly into either $\gamma$ matrix or the $\gamma^{\prime}$ precipitate, see Figs. $1 \mathrm{~b}$ and c. Once the precipitate shape is determined, it can be filled with atoms arranged according to the crystallographic structure and orientation of the lattice of the precipitate phase which - in case it cannot be determined from the APT dataset itself - has to be determined by complementary experiments, e.g. by correlative TEM, electron back-scatter diffraction (EBSD) or X-ray diffraction [41]. The remainder of the sample is filled with atoms on lattice positions of the matrix phase. The approach of using complementary information on the crystallography renders the lattice rectification step $[37,36]$ in the sample reconstruction unnecessary. Atoms of both phases can be furthermore replaced in a stochastic manner according to the local elemental concentrations of $\mathrm{Ni}$ and $\mathrm{Al}$ as determined from APT (Fig. 1e). The resulting structures can in principle be further refined by MC calculations to reproduce the local SRO $[35,38]$. In the current work, $\mathrm{Re}$ and $\mathrm{Al}$ ions were used to define the IPB location since they are mostly found in the $\gamma$ matrix and $\gamma^{\prime}$ particles, respectively (Fig. 1b). The iso-density surfaces were constructed by means of a marching cubes algorithm $[42,43]$ as implemented in the $\mathrm{R}$ statistical software [44]. The number of grid lines to compute the iso-density surfaces was chosen so as to obtain a smooth faceting of the computed surfaces. Fig. 1(c) shows the iso-density surfaces for Re and Al, defined by the location of $70 \%$ of all Re ions and $22.5 \%$ of all $\mathrm{Al}$ ions, respectively. The chosen iso-levels for the two ions provide nearly identical surface topology at the interface. The iso-density surface of $\mathrm{Al}$ was used merely to verify the correctness of the interface predicted by the iso-density surface of Re. For the construction of the atomistic simulation samples from the APT data sets, however, only the iso-density surface of Re was used so as to have a unique definition of the interface. It must be pointed out that the iso-density surface of Re shows negligible differences for iso-levels between $70 \%$ and $90 \%$. Following the generation of the isodensity surfaces, a cuboidal box that represents the desired simulation sample was cut out (Fig. 1c). Care was taken to avoid boundary artifacts whilst preserving topologically relevant features of the APT sample. The atomistic sample (Fig. 1d) was then generated using nanoSCULPT [45, 46]. This tool uses a point in polyhedron test [47] to determine if an atom is inside or outside a volume bounded by an arbitrary surface. In our case the bounded volume corresponds to the $\gamma$ matrix and is defined by the iso-density surface of Re and the sides of the cuboidal box. In a first stage only the region pertaining to $\gamma$ channel is filled with $\mathrm{Ni}$ atoms in a fcc structure, and in a second step, the regions corresponding to the $\gamma$ ' phase are filled with $\mathrm{Ni}_{3} \mathrm{Al}$ in a $\mathrm{L}_{2}$ structure. The lattice orientation corresponds to that of the original single crystal from which the APT-sample was extracted, and the lattice parameters of the two phases were obtained from the embedded atom method (EAM) potentials of Mishin [48]. For pairs of atoms across the curved IPB which were spaced less than $0.45 \cdot a_{0}$ apart, where $a_{0}$ is the lattice constant, one atom at a time was removed in an iterative procedure following the approach described in $[49,26]$.

A second APT-informed sample (Fig. 1e) was generated by stochastically replacing atoms in the original atomistic simulation sample, so as to match the local concentrations of $\mathrm{Al}$ and $\mathrm{Ni}$ as observed in the actual APT data set. The local concentrations were obtained by a voxelised discretisation of the APT data. A voxel size of $1.2 \mathrm{~nm}$ was chosen so as to avoid large fluctuations with neighbouring voxels. The simulation box has dimensions of $45 \times 45 \times 75 \mathrm{~nm}^{3}$, and contains approximately 14 million atoms. As the focus of this study is on fundamental dislocation processes rather than on short-range order effects, we forgo further refinement of the atomic distribution by MC calculations.

\subsection{Molecular dynamics simulations}

Atomistic simulations of the $\gamma / \gamma^{\prime}$ microstructure were performed using the Embedded Atom Method (EAM) potential developed by Mishin [48], which has been shown to represent well the equilibrium properties and defects of both the $\gamma$ and $\gamma^{\prime}$ phases. In addition to the samples generated from the APT data (Fig. 2a and b), two typical idealized samples were used. The setup in Fig. 2(c) has the same dimensions as the APT-informed sample, albeit with a flat (100) IPB instead of the curved precipitate. The geometry and 
(a)

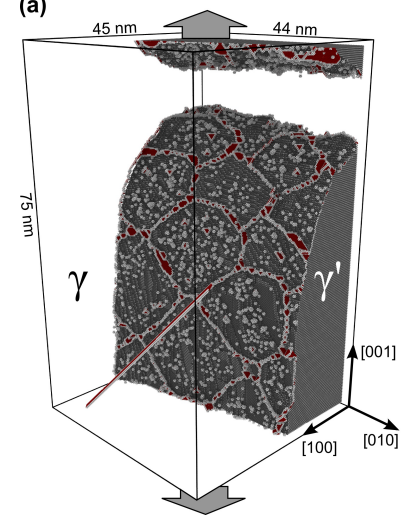

(b)

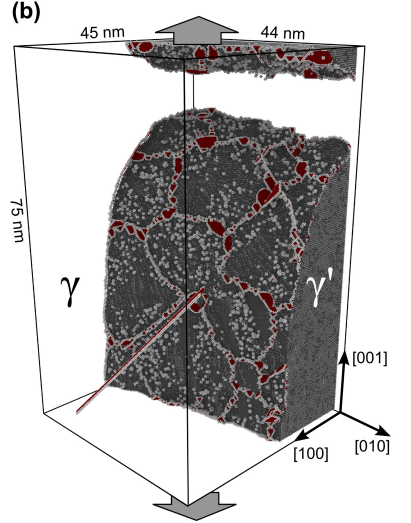

(c)

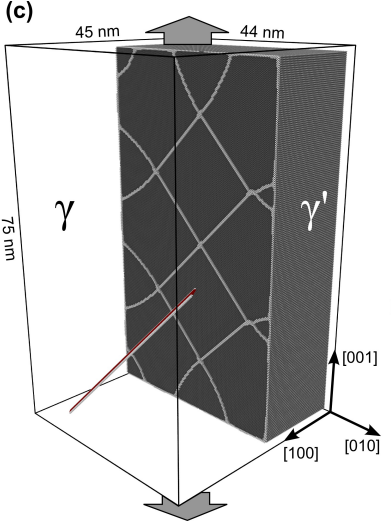

(d)

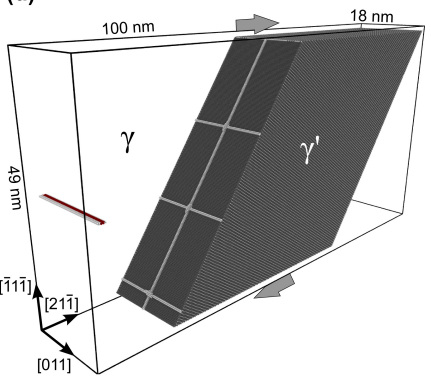

Figure 2: Simulation setups. (a-b) APT-informed simulation samples including one $60^{\circ}$ matrix dislocation. Only atoms belonging to the $\gamma^{\prime}$ phase or defects are shown. (a) sample with stoichiometric $\mathrm{Ni} / \mathrm{Ni}_{3} \mathrm{Al}$ composition, (b) distribution of $\mathrm{Ni}$ and $\mathrm{Al}$ according to the local chemical composition as determined by APT. (c) Atomistic sample with same orientation, size and boundary conditions as (a)\&(b), however with a planar $\gamma / \gamma^{\prime}$ boundary. (d) Quasi-2D slab geometry for force-controlled studies with PBC in dislocation line and propagation direction. The grey atoms belong to the $\gamma^{\prime}$ phase, the red and white atoms, respectively, denote stacking fault and other defect atoms in the $\gamma$ phase, including atoms in dislocation cores or next to vacancies. The loading directions are indicated by grey arrows.

crystallographic orientation match the ones used to study misfit dislocation networks in $[17,19,21]$. However, we use fixed boundary conditions instead of PBC to mimic the situation in the APT-informed sample and to allow for the insertion of matrix dislocations. The fourth scenario, Fig. 2(d), is similar to the one suggested

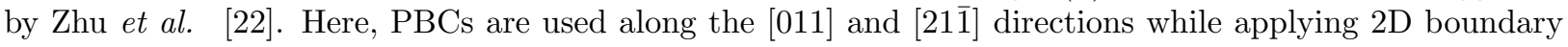
conditions on the surfaces parallel to the glide plane, see Fig. 2(e). In contrast to the approach used by Zhu et al. [22], this setup allows study the interactions of infinite straight screw as well as $60^{\circ}$ dislocations with a planar $\{100\}$ IPB in a controlled shear stress state.

Dislocations were inserted in the simulation boxes following the procedure described in [50]. All structures were optimized using the FIRE algorithm [51]. The lattice is then expanded according to the average lattice constant of the $\gamma / \gamma^{\prime}$ microstructure at $1250 \mathrm{~K}$, and equilibrated at $1250 \mathrm{~K}$ for at least 80 ps while maintaining zero stresses using a Nosé-Hoover-type thermo- and barostat [52]. The cuboidal simulations boxes with fixed boundary conditions are loaded by homogeneously scaling the atomic positions according to a uniaxial strain rate of $\dot{\epsilon}=10^{8} \mathrm{~s}^{-1}$ along the [001] direction, while maintaining constant temperature and zero stress in the orthogonal directions.

The dislocation in the slab sample was loaded by force boundary conditions on the surfaces parallel to the glide plane and in Burgers vector direction, at a shear stress rate $\dot{\tau}=4 \cdot 10^{9} \mathrm{GPa} \mathrm{s}^{-1}$ equivalent to the strain rate in the other samples. To eliminate thermal fluctuations, snapshots were obtained by averaging atomic positions over $300 \mathrm{fs}$. Defect structures were identified using AtomViewer [53, 54] and visualized with OVITO [55].

\section{Results}

\subsection{Interphase boundary and misfit dislocation network}

Due to the positive lattice misfit $\delta$ between $\mathrm{Ni}$ (lattice constant $\left.a_{0}(\mathrm{Ni})=0.352 \mathrm{~nm}\right)$ and $\mathrm{Ni}_{3} \mathrm{Al}\left(a_{0}\left(\mathrm{Ni}_{3} \mathrm{Al}\right)=0.3571\right.$ $\mathrm{nm})[48]$ of $\delta=2 \frac{a_{0}\left(\mathrm{Ni}_{3} \mathrm{Al}\right)-a_{0}(\mathrm{Ni})}{a_{0}\left(\mathrm{Ni}_{3} \mathrm{Al}\right)+a_{0}(\mathrm{Ni})} \cdot 100 \%=1.45 \%$, a misfit dislocation network is automatically formed upon energy minimization. The structure of the resulting misfit dislocation network in the different samples at $1250 \mathrm{~K}$ is shown in Figs. 3a-d. A perfectly rectangular network (Fig. 3d) is formed in the quasi-2D sample with constricted edge dislocations with Burgers vector $\vec{b}=a_{0} / 2\langle 011\rangle$ lying along the [011] and [01 $\left.\overline{1}\right]$ directions. The fixed boundary conditions used in the 3D sample with planar IPB lead to some deviations from 
the rectangular network (Fig. 3c), but overall the same dislocation line directions and Burgers vectors are maintained as in the $2 \mathrm{D}$ sample. At some of the intersections between the misfit dislocations, short segments with $\langle 001\rangle$ line direction and $\vec{b}=a_{0} / 2\langle 010\rangle$ are formed. The misfit dislocations in the APT-informed simulation samples, Figs. 3a,b, however, show clear deviations from the rectangular network structure, and include dislocation segments with Burgers vectors along [110] and [101] directions pointing out of the mostly (100)-oriented IPB. These dislocations also form square like structures at the intersection between dislocations with Burgers vectors [011] and [011]. The misfit dislocation network mimics the interfacial dislocation network formed during the initial stages of high temperature, low stress creep under $\langle 001\rangle$ loading, see e.g. $[8,56,57,58]$, and contains the same Burgers vectors and line directions.

A particularity of the APT-informed simulation samples is that many of the misfit dislocations are not constricted like in the samples with planar IPB. Instead, the dislocations are dissociated into Shockley partial dislocations on the $(b)$ and $(d)$ planes (Thompson tetrahedron notation [59]), see Figs. 3e-g. We observe two types of dissociation reactions, namely simultaneous dissociation on two $\{111\}$ planes intersecting along the dislocation line where the Shockley partial dislocations are connected by a $a_{0} / 6\langle 110\rangle$-type stair-rod dislocation in a Lomer-Cottrell (LC) [59] lock-like configuration (see Fig. 3e), and splitting on one $\{111\}$ plane which is a local tangential plane to the IPB. The Shockley partial dislocations on parallel $\{111\}$ planes are connected by either, a set of $a_{0} / 6\langle 110\rangle$-type stair-rod dislocations or $a_{0} / 3\langle 100\rangle$-type Hirth dislocations, see Figs. 3f,g. Interestingly, for the APT-informed sample which takes into account the local $\mathrm{Ni}$ and $\mathrm{Al}$ distribution, more misfit dislocations are split into partial dislocations, and the overall number of atoms in stacking fault positions is approximately $25-30 \%$ higher than in the stoichiometric atomistic simulation sample composed of pure $\mathrm{Ni}$ and $\mathrm{Ni}_{3} \mathrm{Al}$, see Fig. 3(b).

During the generation of the atomistic simulation samples from the APT data, atoms that are located very close to each other across the curved IPB are removed using the same criteria as to eliminate excess atoms at grain boundaries in simulations of polycrystalline samples $[49,26]$. This leads to an increase in the density of vacancies near the interface, which can rearrange into stacking fault tetrahedra (SFT), see Figs. 3a,b.

\subsection{Interactions between a single matrix dislocation and the misfit dislocation network}

Under a tensile load in [001] direction, typical of turbine blade applications, only $60^{\circ}$ and screw dislocation segments are deposited along the $\{100\}$ IPB. Dislocations which would deposit segments of other character at the IPBs do not experience any resolved shear stress. The interaction of infinite screw dislocations with the misfit dislocation network was recently studied by Zhu et al. [22] in a 2D setup at 0K. They found that screw dislocations are absorbed in the misfit dislocation network, and a dislocation segment is only deposited when the screw dislocation intersects the misfit dislocation network exactly at the location of a misfit dislocation, with which it reacts [22]. Our results on screw dislocations in the $2 \mathrm{D}$ setup agree qualitatively with their observations, see Supplementary Fig. 1, and we hence focus on the interaction of $60^{\circ}$ dislocations with the misfit dislocation network at a constant temperature of $1250 \mathrm{~K}$. In the following, the interactions of matrix dislocations with the misfit dislocation network are presented only for the samples with stoichiometric $\mathrm{Ni} / \mathrm{Ni}_{3} \mathrm{Al}$ composition. The APT-informed simulation sample with adjusted local elemental concentration shows the same mechanisms.

A $60^{\circ}$ dislocation with $\vec{b}=a_{0} / 2[\overline{1} 01]$ (or $C B(a)$ in Thompson tetrahedron notation [59]) is initially inserted as a straight line in [011] direction in the APT-informed samples and the 3D sample with planar IPB. This dislocation will in the following be referred to as $d_{1}$. The dislocation $d_{1}$ is pinned at the fixed surfaces of the sample and starts to bow out towards the $\gamma^{\prime}$ phase once the sample is deformed under uniaxial tension along the [001] direction. The deposition process of $\mathrm{d}_{1}$ at the IPB of the APT-informed sample is shown in Fig. 4(a) and Supplementary Movie M1. While during the deposition process $\mathrm{d}_{1}$ just intersects the orthogonal misfit dislocations with Burgers vector $D C$, see Fig. $4 \mathrm{~b}$ and Supplementary Fig. 2, it reacts with the orthogonal misfit dislocation $C B$. The details of this process are shown in Fig. 4(d). Both dislocations have the same Burgers vector, and $d_{1}$ is joined to the misfit dislocation on one side, while the threading part of $\mathrm{d}_{1}$ becomes connected to the misfit dislocation on the other side, leaving a gap between the deposited dislocation segments (see also Supplementary Fig. 3). In single-phase fcc metals, this type of interaction among dislocations of same Burgers vectors on intersecting glide planes is referred to as a 
(a)

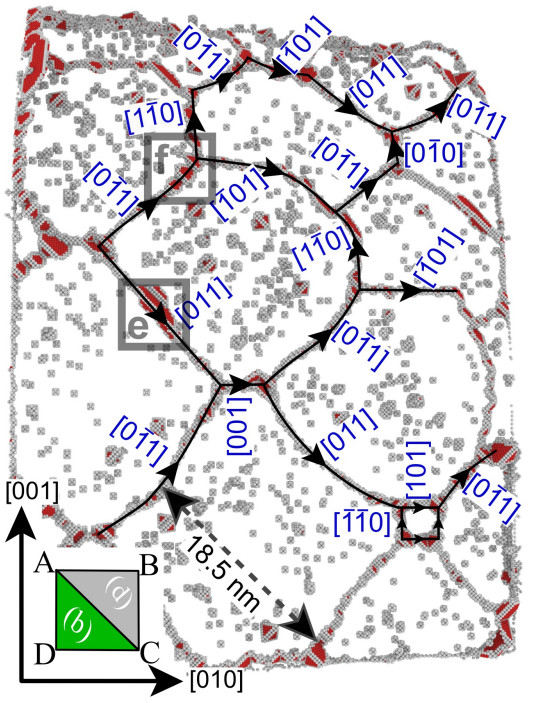

(e)

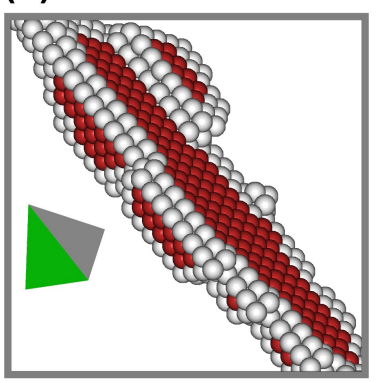

(f)
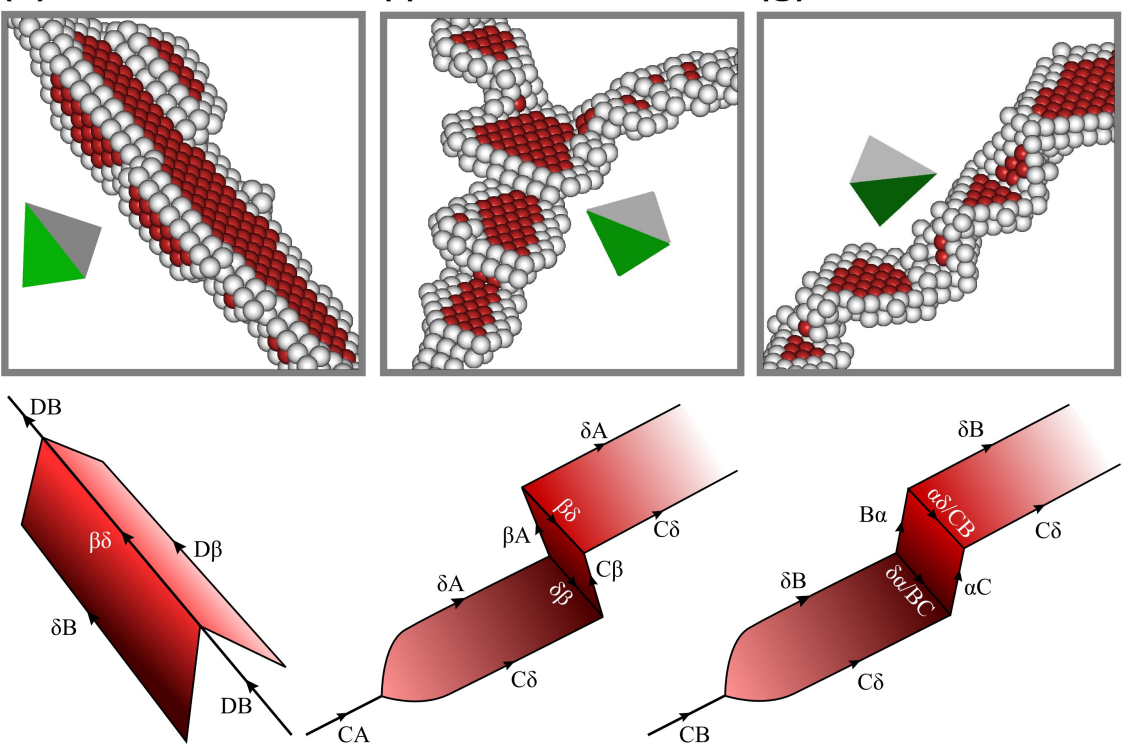

(c)

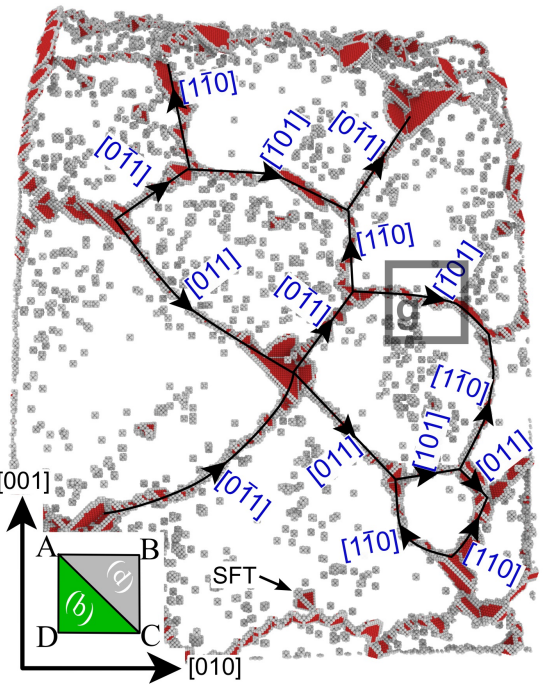

(g)

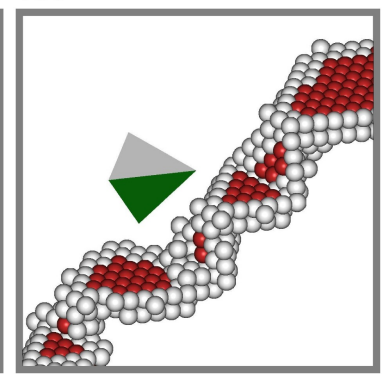

(d)

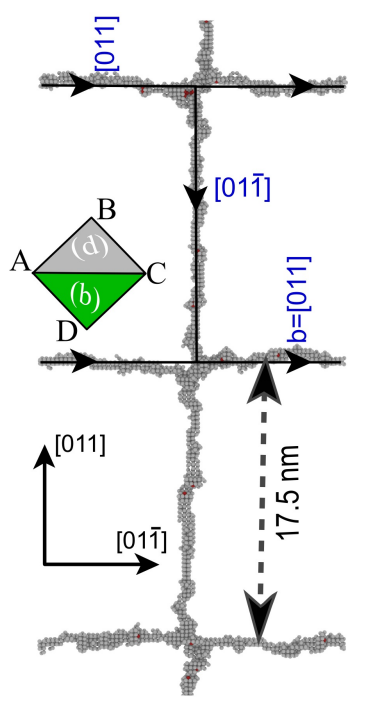

Figure 3: Structure of the misfit dislocation networks and core structure of misfit dislocations. (a),(b) Misfit dislocation network on the $\gamma / \gamma^{\prime}$ interface in the APT-informed simulation samples, with (a) stoichiometric (pure Ni/Ni $\mathrm{Al}_{3}$ ) and (b) nonstoichiometric chemical composition according to the local chemical composition as determined by APT. More stacking faults can be observed in the non-stoichiometric sample. Both samples exhibit large number of vacancies, which sometimes rearrange to form stacking fault tetrahedra (SFT). (c) Misfit dislocation network in the 3D sample with planar interface. (d) Misfit dislocation network in the quasi-2D slab sample. (e)-(g) Typical dislocation core structures of the misfit dislocations in (a),(b) and their schematic representation together with the Burgers vectors in Thompson tetrahedron notation. Burgers vectors are noted in blue, black arrows indicate the line direction of the dislocations. The Thompson tetrahedron indicates the orientation of the corresponding sample. See Fig. 2 for the colouring scheme of the atoms. 
collinear interaction [60]. This kind of interaction is not observed in the 3D sample with planar IPB, as there are no misfit dislocation segments that have the same Burgers vector as $\mathrm{d}_{1}$; only the intersection process is observed, which is described in more detail in Supplementary Fig. 4 and movie M2.

Compared to the $3 \mathrm{D}$ samples, the interaction of a straight infinite $60^{\circ}$ dislocation $C B(d)$ (which is crystallographically equivalent to $\mathrm{d}_{1}$ used in the $3 \mathrm{D}$ samples) with the misfit dislocation network is completely different in the quasi-2D setup. Here, the misfit dislocations are completely free to move within the IPB plane. The misfit dislocation $B D$ parallel to $\mathrm{d}_{1}$ and below its glide plane moves upwards and reacts with $\mathrm{d}_{1}$ at its intersection point with the network. The reaction leads to a dislocation $D C$ that is dissociated on the (b) plane, see Supplementary Fig. 5 and movie M3. By contrast, no significant glide of the misfit dislocations along the IPB can be observed in the 3D samples. This can be attributed to the fixed boundary conditions in the 3D samples, and the fact that a larger network is simulated rather than just a periodically repeating unit.

When $d_{1}$ in the APT-informed simulation sample approaches the edge of the precipitate, a second collinear interaction with an orthogonal misfit dislocation takes place, see Fig. 4(e). However, as $d_{1}$ can no longer deposit segments at the IPB, it is no longer connected to the precipitate. The misfit dislocation to which $\mathrm{d}_{1}$ is connected is therefore knitted-out from the misfit dislocation network. Furthermore, it is interesting to note that during the deposition of $\mathrm{d}_{1}$ at the IPB between two misfit dislocations, the dislocation absorbs vacancies, leading to local climb of $\mathrm{d}_{1}$, see Fig. 4(c) and Supplementary Movie M4.

\subsection{Cutting of the $\gamma^{\prime}$ phase by a pair of matrix dislocations}

To study the cutting of the $\gamma^{\prime}$ precipitate by a pair of $60^{\circ}$ dislocations, two straight dislocations with identical Burgers vector $C B(a)$ are inserted on the same glide plane $6 \mathrm{~nm}$ apart. During relaxation and thermalization, the first and leading dislocation, $\mathrm{d}_{1}$, reacts to the stress field of the second dislocation $\left(\mathrm{d}_{2}\right)$ by gliding towards the precipitate and depositing dislocation segments at the IPB similar to the deposition of the single $\mathrm{d}_{1}$ described in the previous section. The resulting configuration at zero applied strain is shown in Fig. 5(a).

The overall evolution under uniaxial strain is shown in Fig. 5(a) and Supplementary movie M5. With increasing strain, $\mathrm{d}_{1}$ proceeds to deposit itself on the IPB; $\mathrm{d}_{2}$ follows $\mathrm{d}_{1}$ and bows out towards the IPB, see Fig. 5(a). With additional strain the parts of $\mathrm{d}_{1}$ between the misfit dislocations cut into the $\gamma^{\prime}$ phase. Cutting of the $\gamma^{\prime}$ phase by the leading partial dislocation $C \alpha$ of $\mathrm{d}_{1}$ results in a complex stacking fault (CSF), which is transformed into an antiphase boundary (APB) by the trailing partial dislocation $\alpha B$ of $\mathrm{d}_{1}$, see Fig. 5(b). Where $\mathrm{d}_{2}$ glides on the identical plane as $\mathrm{d}_{1}$, it also cuts the $\gamma^{\prime}$ phase where the leading partial dislocation of $\mathrm{d}_{2}$ forms another CSF while the trailing partial dislocation restores the ordered $\mathrm{L} 1_{2}$ crystal structure of the $\gamma^{\prime}$ phase. Arranged jointly on the same glide plane, the two inserted dislocations form a classical superdislocation, see Fig. 5(b). Superpartial dislocations in $\gamma^{\prime}$ are in the following denoted by $\mathrm{d}^{\prime}$.

Where $\mathrm{d}_{1}$ intersects the misfit dislocation network, two characteristic processes occur. The intersected orthogonal misfit dislocations are dissociated into partial dislocations on the $(b)$ and $(d)$ planes linked by the stair-rod dislocation $\delta \beta$, as described in Fig. 3(e). The partial dislocation $B \delta$ of the misfit dislocation is connected to $\mathrm{d}_{1}$ (Burgers vector $C B(a)$ ) together with a second partial dislocation $C \delta$ which was generated during the intersection process, see Supplementary Figs. 6a-d. Under the stress field of the approaching $\mathrm{d}_{2}$, this dislocation $C B(d)$ enters the $\gamma^{\prime}$ phase together with $\mathrm{d}_{1}$; however, on the cross-slip plane. In other words, the intersection of the misfit dislocation network by the $60^{\circ}$ dislocation can activate dislocations on a second glide plane within $\gamma^{\prime}$, as shown schematically in Fig. 5(e). The created superpartial dislocation is still connected to the orthogonal misfit dislocation at the IPB. This process is also observed in the 3D sample with planar IPB, but only at high strains, where the initially constricted misfit dislocations dissociate into the LC-lock structure described in Fig. 3(e), see Supplementary Fig. 7 and movie M6.

When $\mathrm{d}_{2}$ arrives at the intersection of $\mathrm{d}_{1}^{\prime}$ with the misfit dislocation network, the interaction of $\mathrm{d}_{2}$ with the intersection of $\mathrm{d}_{1}^{\prime}$ and the misfit dislocation network leads to the depinning of $\mathrm{d}_{1}^{\prime}$ after a series of intermediate reactions and the pinning of $\mathrm{d}_{2}$ at the misfit dislocation (see Supplementary Fig. 6 and movie M7 for more details). Alternatively, $\mathrm{d}_{1}^{\prime}$, which is pinned at an misfit dislocation, can bow out on both sides of this misfit dislocation and depin in an Orowan-type mechanism, see Fig. 5(c). This process is also observed in the 3D sample with planar IPB, see Supplementary Movie M8. 
(a)

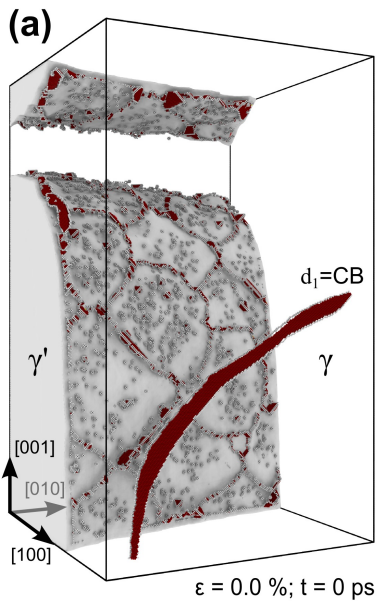

(b)

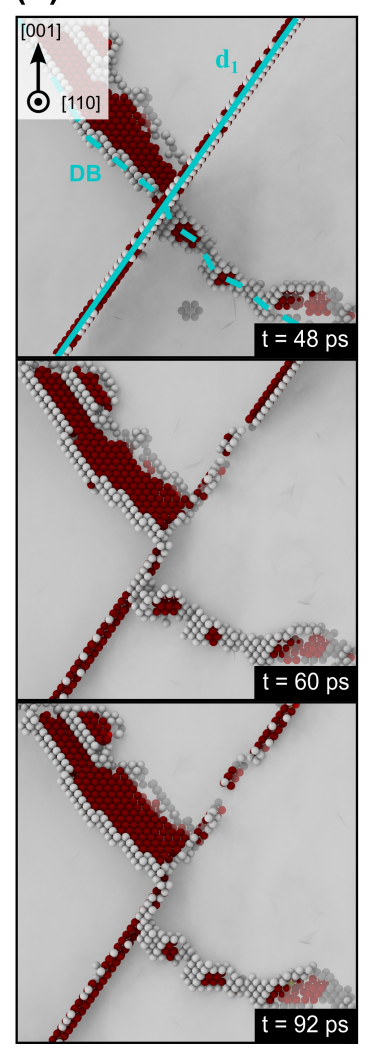

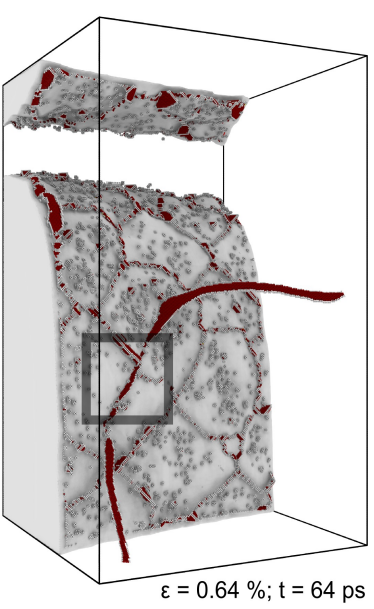

(c)

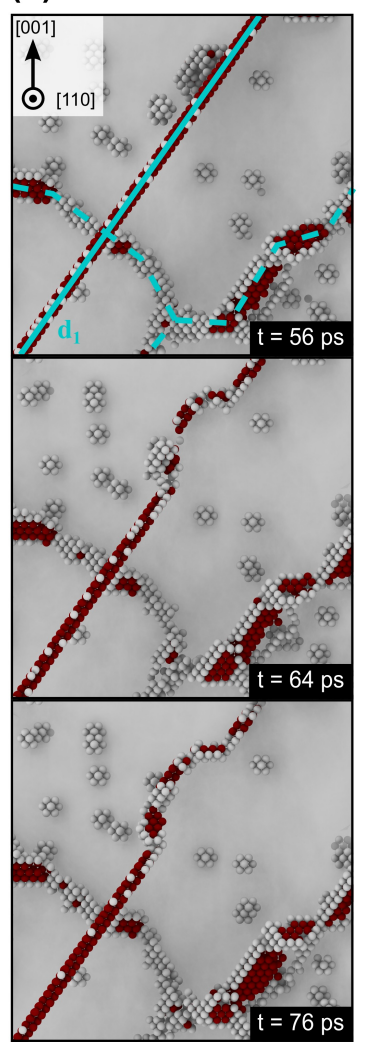

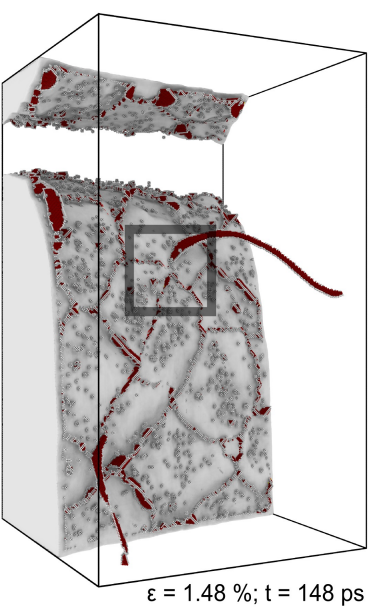

(d)

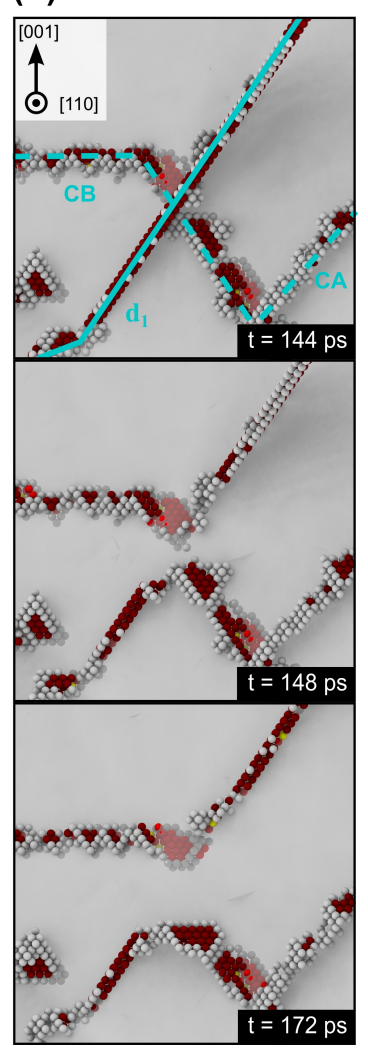

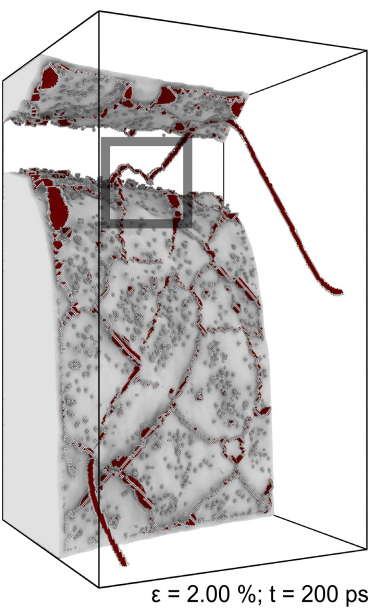

(e)

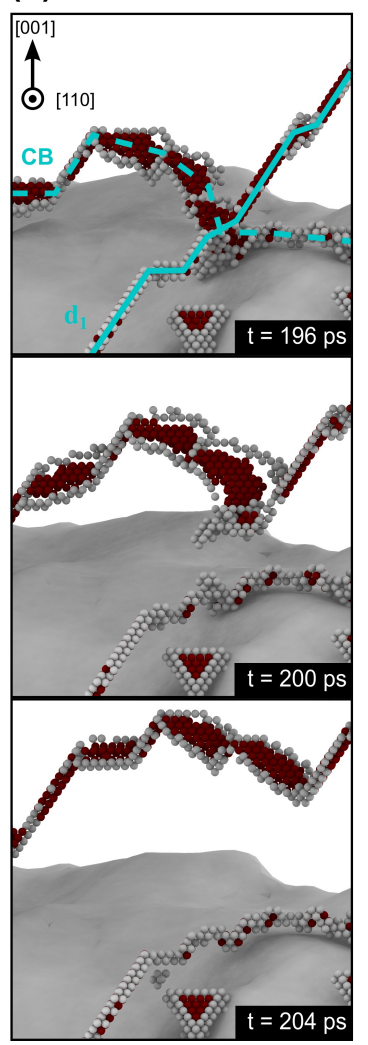

Figure 4: Interaction of one $60^{\circ}$ matrix dislocation $d_{1}$ with the misfit dislocation network in the APT-informed sample. (a) Deposition of dislocation segments at the interface during tensile loading in [001] direction. (b)-(d) Details of specific dislocation processes which are highlighted by grey rectangles in (a). (b) Intersection of an orthogonal misfit dislocation by the deposited $d_{1}$. (c) Local climb of $d_{1}$ by absorption of vacancies at the interface. (d) collinear reaction of $d_{1}$ with a misfit dislocation of identical Burgers vector leaving a gap along the line of the deposited dislocation. (e) Knitting-out of a misfit dislocation by $\mathrm{d}_{1}$. Defects as identified by AtomViewer [53,54]: red - stacking fault (lighter shade used to denote complex stacking fault (CSF) in $\gamma^{\prime}$ phase), white - other defects. To improve clarity, undercoordinated atoms next to vacancies were removed from the pictures in (b),(d),(e). The $\gamma / \gamma^{\prime}$ interface is indicated by a semi-transparent surface. 
The situation is different where the collinear reaction of $d_{1}$ with the misfit dislocation led to a gap along the deposited dislocation line. Here, $\mathrm{d}_{1}^{\prime}$ remains pinned at the IPB where it is connected to the orthogonal misfit dislocation, see the illustration in Fig. $5(\mathrm{~d})$. The threading dislocation $\mathrm{d}_{2}$ interacts with this dislocation arrangement resulting in a series of collinear reactions similar to that observed in the case of $\mathrm{d}_{1}$. In the process $\mathrm{d}_{1}^{\prime}$ unpins from the misfit dislocation and becomes connected to the threading second matrix dislocation in the $\gamma$ phase, $\mathrm{d}_{2}$. The segment of $\mathrm{d}_{2}$ deposited along the IPB cuts into the $\gamma^{\prime}$ phase forming the trailing superpartial dislocation $\mathrm{d}_{2}^{\prime}$, which is now pinned at the misfit dislocation. Fig. 5(b) shows this configuration where $\mathrm{d}_{2}$ has become connected to $\mathrm{d}_{1}^{\prime}$. The process is described in more detail in the Supplementary Fig. 8 and movie M9.

In contrast to the above, in the $2 \mathrm{D}$ setup, the stress field of $\mathrm{d}_{2}$ leads to the emission of the dislocation $D C(b)$ - result of the reaction of $\mathrm{d}_{1}$ with the parallel misfit dislocation - back into the $\gamma$ phase, see Supplementary Fig. 9.

It is interesting to note that the local climb due to the absorption of vacancies by $\mathrm{d}_{1}$ in the APT-informed samples leads to jogs in $d_{1}^{\prime}$. In the $\gamma^{\prime}$ phase these jogs lead to steps in the APB. The jog segments of $d_{1}^{\prime}$ which are on different planes can merge together leaving behind prismatic loops that collapse to vacancy clusters at the end of the steps, see the black arrow in Fig. 5(b). This mechanism, which effectively reverses the climb process, is described in more detail in Supplementary Fig. 10. Where $\mathrm{d}_{1}$ has absorbed vacancies, the glide planes of $\mathrm{d}_{1}$ and $\mathrm{d}_{2}$ are locally no longer identical. Therefore, $\mathrm{d}_{2}$ can enter the $\gamma^{\prime}$ particle on slip planes adjacent to the one of $\mathrm{d}_{1}^{\prime}$, which leads to the situation where stacking faults are created on neighbouring planes, as indicated by the white arrow in Fig. 5(b).

\section{Discussion}

Two key observations can be made from the simulations of matrix dislocations interacting with a semicoherent precipitate in the four different computational setups: (a) the misfit dislocation network and the core structure of misfit dislocations are critically influenced by the simulation setup, and in particular by the local curvature of the IPB; (b) the nature of the misfit dislocations significantly affects the interaction of matrix dislocations with the misfit dislocation network.

The differences in the topology of the misfit dislocation network in the APT-informed samples compared to the perfectly rectangular network in the quasi-2D sample and the nearly perfectly rectangular network in the 3D sample with planar IPB, Figs. 3a-d, is caused by several factors. On the curved IPB of the APT-informed samples the dislocations can rearrange to shorten their line length. This is impossible in the quasi-2D sample with PBC, and in the 3D sample with planar IPB only observed near the fixed borders, see Fig. 3(c). Furthermore, the misfit dislocation network in the APT-informed sample contains edge dislocations with Burgers vectors of the type $[ \pm 10 \pm 1]$ which point into or out of the (100) plane. Although the (100) plane is the average IPB plane along most of the precipitate, locally the IPB intersects (100) lattice planes. Here misfit dislocations can form with a Burgers vector component according to the misfit in the [100] direction (Fig. 6a).

In addition to the misfit dislocation network topology, the core structure of the misfit dislocations in the APT-informed simulation sample also differs significantly from the misfit dislocations in the samples with planar IPB. In general, perfect $a / 2\langle 110\rangle$-type dislocations in fcc structures dissociate into Shockley partial dislocations with $a / 6\langle 112\rangle$ Burgers vectors to lower their strain energy according to Frank's rule [59]. However, this is only possible on $\{111\}$ glide planes where the generalized stacking fault energy surface has a local minimum at the intrinsic stacking fault configuration. The dislocations on the planar (100) IPBs in the Figs. 3c,d can therefore not dissociate. In the APT-informed samples the local curvature of the IPB causes misfit dislocation segments to lie on tangential $\{111\}$ planes, where they can dissociate, see Fig. 6(b). Stair-rod or Hirth dislocations then connect the dissociated dislocations on the adjacent tangential planes, see Figs. 3f,g. Misfit dislocations which are oriented along a $\langle 110\rangle$ direction can, furthermore, dissociate simultaneously on two $\{111\}$ planes connected by a stair rod dislocation, forming a LC-lock, see Fig. 3(e). According to Frank's rule, this configuration has still about 30\% lower strain energy than a perfect dislocation. This is, however, only possible if the misfit dislocation has a certain standoff to the local IPB plane - otherwise the partial dislocations would cut into the $\gamma^{\prime}$ phase creating a CSF. This is why 
(a)

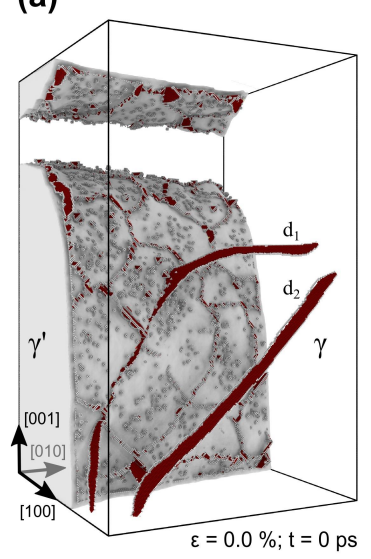

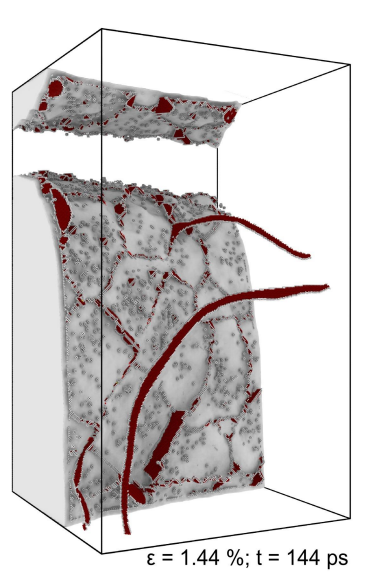
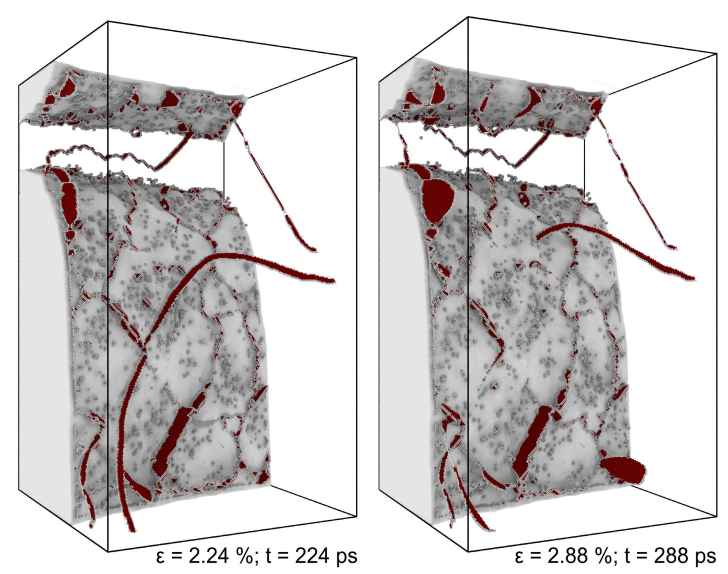

(b)

(c)

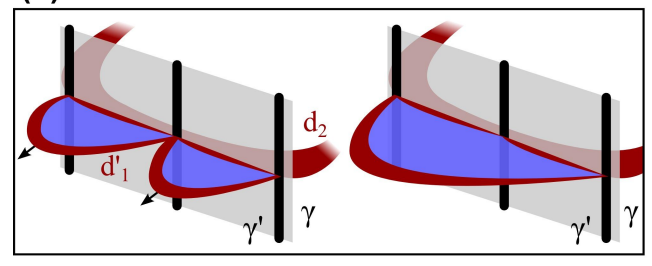

(d)

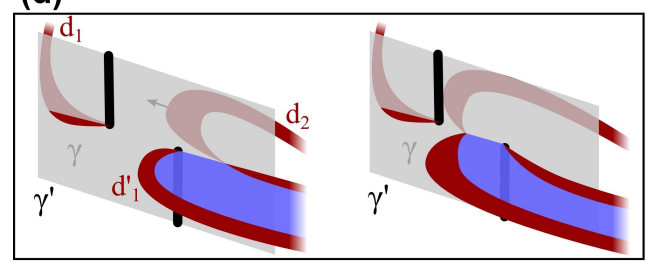

(e)

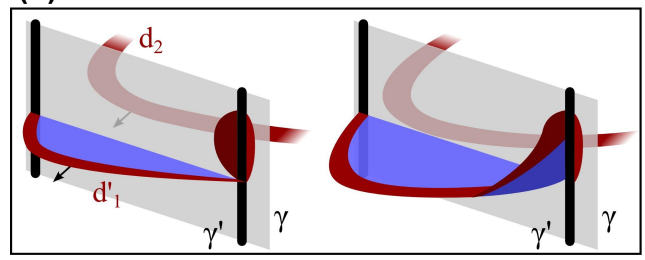

(f)

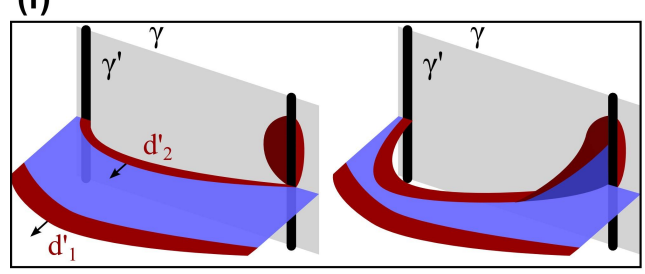

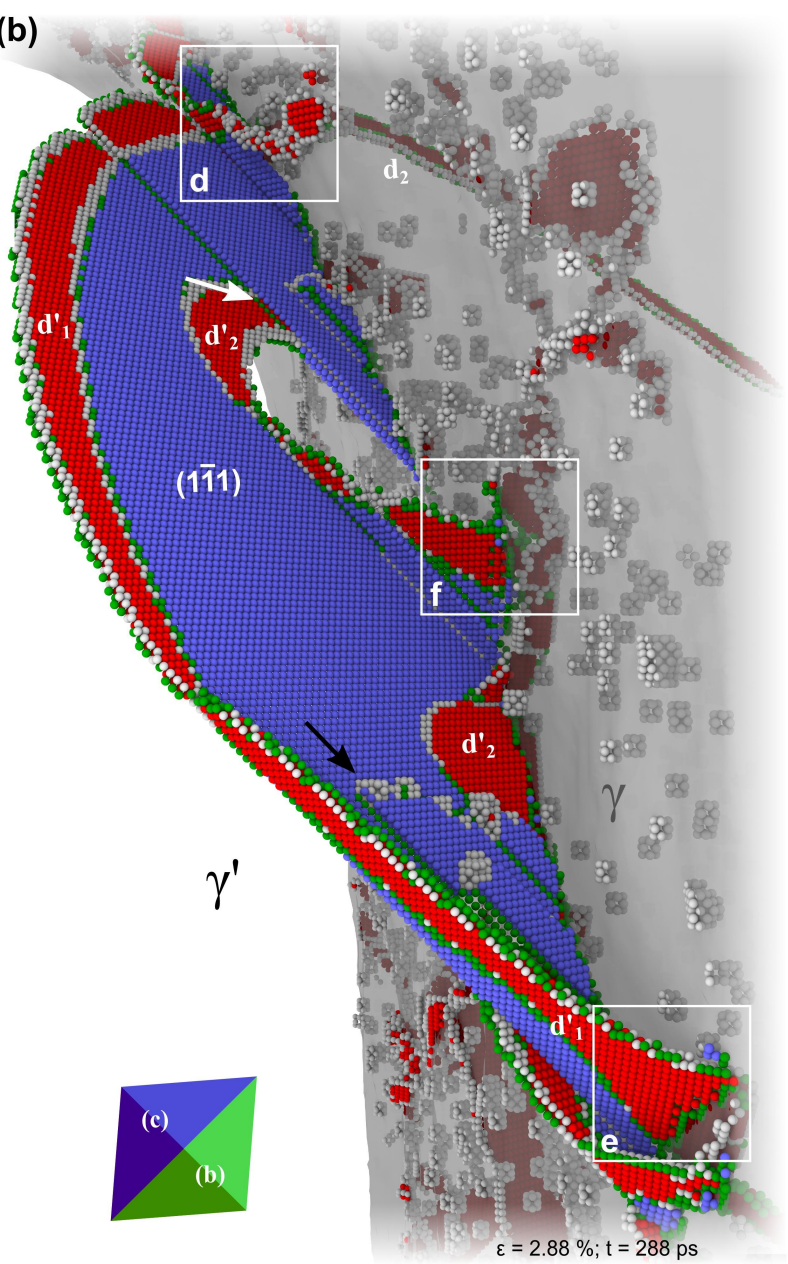

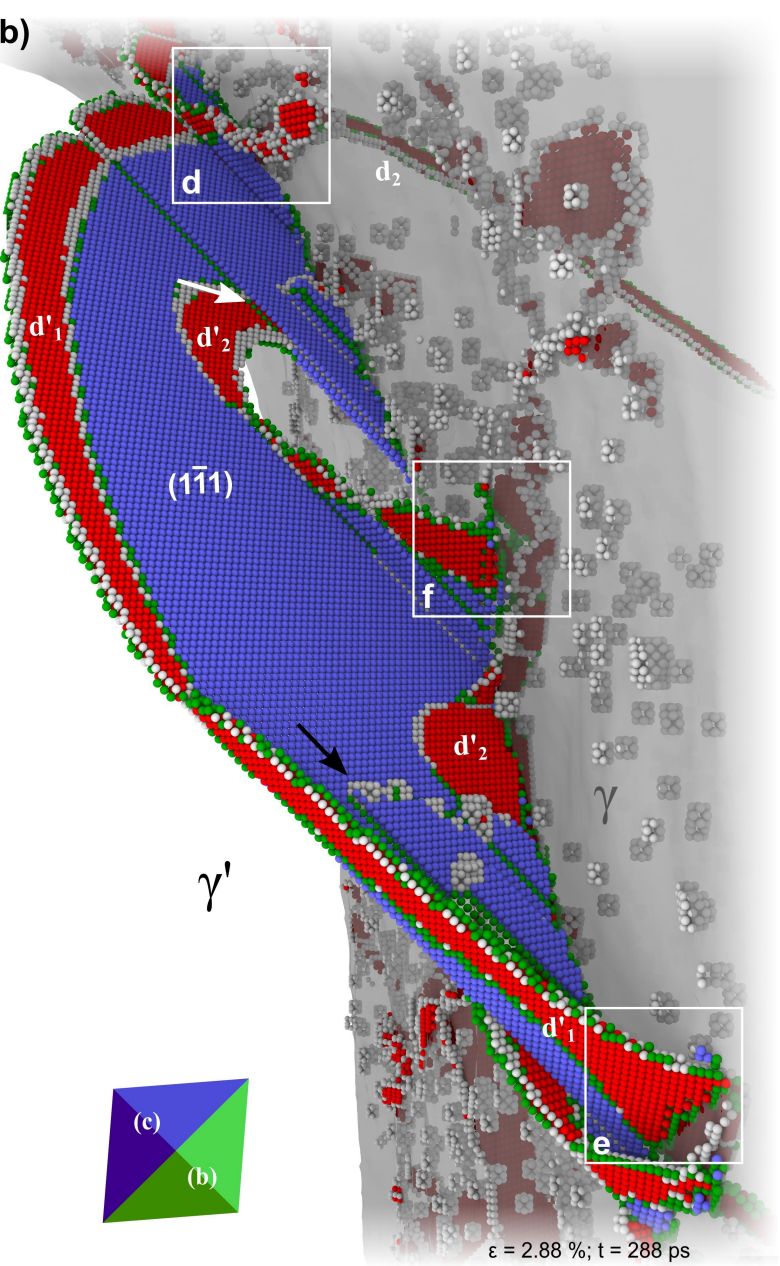

Figure 5: Interaction of two $60^{\circ}$ matrix dislocations, $\mathrm{d}_{1}$ and $\mathrm{d}_{2}$, with the misfit dislocation network in the APT-informed sample. (a) Deposition of dislocation segments at the interface during tensile loading in [001] direction. (b) Details of the last configuration of (a), showing the formation of a superdislocation in the $\gamma^{\prime}$ phase. The white arrow indicates where, due to the jogged $\mathrm{d}_{1}^{\prime}$, a stacking fault is produced by $\mathrm{d}_{2}^{\prime}$ on an plane adjacent to the existing APB. The black arrow indicates steps in the APB and prismatic loops left behind by the motion of a jogged segment $\mathrm{d}_{1}^{\prime}$, as described in more detail in the text and in Supplementary Fig. 10. (c-f) Idealized sketches of the different processes which lead to the configuration in (b). The grey plane represents the $\gamma / \gamma^{\prime}$ interface, the black line the misfit dislocations, red represents stacking faults, blue represents the antiphase boundary. See text as well as Supplementary Figs. 6,8 and movies M5, M7-M9 for more details. (c) Depinning of a superdislocation from a misfit dislocation by an Orowan-like process. (d) The collinear interaction described in Fig. 4(d) left a gap in the deposited dislocation line. The second matrix dislocation $\mathrm{d}_{2}$ can thus not cut into the $\gamma^{\prime}$ phase, but can unpin the leading superpartial dislocation $\mathrm{d}_{1}^{\prime}$ from the misfit dislocation by a collinear reaction during which $\mathrm{d}_{2}$ merges with $\mathrm{d}_{1}^{\prime}$. (e) Reaction of $\mathrm{d}_{1}^{\prime}$ with a partial dislocation of the LC-lock like structure, leading to a superpartial dislocation on the cross-slip plane. (f) Similar mechanism like (e), however for $\mathrm{d}_{2}^{\prime}$. Defects as identified by AtomViewer [53, 54]: red - stacking fault (lighter shade used to denote complex stacking fault (CSF) in $\gamma^{\prime}$ phase), blue - antiphase boundary, green - other 12-neighbors defects, white - other defects. The $\gamma / \gamma^{\prime}$ interface is indicated by a semi-transparent surface. 
this dissociation is observed in simulations with planar IPBs only at high stresses. The overall increase in stacking fault area in the APT-informed sample with adjusted local $\mathrm{Ni}$ and $\mathrm{Al}$ concentrations compared to the stoichiometric sample, Fig. 3(b), can be related to the decrease of the stable stacking fault energy in the $\gamma$ phase with increasing Al concentration, see Supplementary Fig. 11.

A LC-lock like core structure is also seen in the HRSTEM micrograph Fig. 7(b) of a misfit dislocation in a high-temperature crept sample of LEK94 [61] (see Supplementary Information for details on the material and sample preparation). Like all technical Ni-base superalloys, this alloy has a negative lattice misfit resulting in mismatch-accommodating dislocations of opposite Burgers vectors compared to the misfit dislocations in the present simulation study on pure $\mathrm{Ni} / \mathrm{Ni}_{3} \mathrm{Al}$, which has a positive misfit. In order to arrange the Shockley partial dislocations in such a way that an intrinsic stacking fault is formed between them and the stair-rod dislocation, the glide planes on which the dislocations dissociate have to be interchanged [59]. In other words, instead of the dissociation of $D B$ shown in Fig. 3(e), the dislocation of opposite sign, $B D$, would be dissociated in $\beta B(b)$ on the left and $B \delta(d)$ on the right. LC-lock like dissociation of mismatch-accommodating dislocations on planes towards the $\gamma^{\prime}$ phase similar to the one observed in Fig. 7(a) could however be expected in Co-base superalloys, which have a positive lattice misfit [62, 63]. LC-lock like core structures of interfacial dislocations were recently also reported for the alloy DD6 [64] crept under loading conditions different from [61]. The dissociation of misfit dislocations into LC-locks was also observed in atomistic simulations of fcc metal multilayers [65]. Hence we speculate that this type of core structure is rather typical for mismatch-accommodating dislocations in fcc crystals in general, and for the $\gamma / \gamma^{\prime}$ microstructure of superalloys in particular.

While purely rectangular interfacial dislocation networks like in Figs. 3c,d are rarely reported in experiments, the misfit dislocation network in the APT-informed samples, Figs. 3a,b, bear striking resemblance to the mismatch network developed during the initial stages of high temperature creep of Ni-base superalloys loaded in [001] direction [8, 56, 57, 58, 66, 64]. The dislocations line directions and Burgers vectors in the misfit dislocation network are of the same type as in the experiments, including Burgers vectors of $\langle 001\rangle$-type [8, 56, 64], Burgers vectors with components normal to the IPB plane and the formation of little square loops between larger structures, see e.g. [8, 57].

The evolution of the dislocation network of deposited glide dislocations during high temperature, low stress creep into a mismatch-accommodating interfacial dislocation network has been studied by many groups $[8,9,5,56,67,68,58,69]$. The formation of a mismatch-accommodating interfacial dislocation network is believed to involve $[8,9,5,56]$ : (a) the reaction between deposited glide dislocations on different activated glide systems, (b) the splitting of the nodes formed during the aforementioned reactions, (c) the re-orientation of dislocation lines in edge orientation, (d) the reaction of the so formed secondary dislocations with further primary glide dislocations. Additionally, local diffusion processes and local climb, but without long-range glide or climb along the interface, also play a role.

Given that the processes by which the mismatch-accommodating interfacial dislocation networks form in experiments are completely different from the automatic formation of a misfit dislocation network by energy minimization of a two-phase structure with different lattice constants, the resemblances between experiments and atomistic simulations on the APT-informed samples might be fortuitous. However, the driving force in both cases is the minimization of strain energy. During high temperature, low stress creep the deposited channel dislocations effectively reduce the misfit strain by increasingly forming edge dislocations with Burgers vectors which are parallel to the IPB plane, see e.g. [58]. By not artificially constraining the misfit dislocation network formation in the simulations to a planar interface with PBCs, other energy minima become accessible during energy minimization, leading, e.g., to different dislocation core structures compared to typical simulation setups. The resulting network structures in the experiments as well as in simulations with realistic IPBs should therefore in both cases be close to an energetic minimum. The misfit dislocation network in the APT-informed sample and the misfit-accommodating interfacial network in experiments can thus be expected to share similar features. This has important consequences for atomistic modelling: in contrast to the typically used overly idealized simulation scenarios, APT-informed atomistic samples indeed provide a more realistic model for interfacial dislocation networks. Furthermore, it is to be expected that also under experiment conditions, the local IPB curvature influences the topology, core structure and formation of misfit-accommodating interfacial dislocation networks. 
(a)

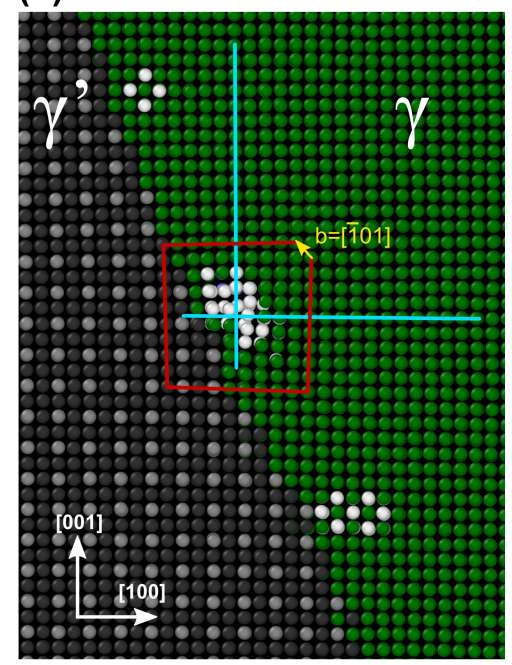

(b)

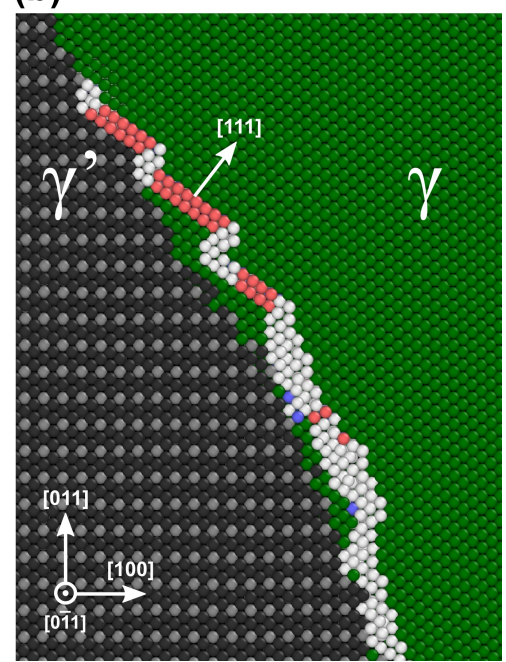

Figure 6: Effect of interface curvature on misfit dislocations. (a) Misfit dislocation with Burgers vector of [101] type (yellow arrow). The local interface plane intersects both the (100) and (001) planes, which allows the additional (001) and (100) half planes (blue lines) due to the different lattice constants to combine into one misfit dislocation. The red line shows the Burgers circuit. (b) Where the interface can be approximated by local (111) tangential planes, the misfit dislocation can dissociate into Shockley partial dislocations on these planes. Colour code: green - fcc structure in the $\gamma$ phase, grey $-\mathrm{L} 1_{2}$ structure in $\gamma^{\prime}$ phase, red - stacking-fault, white - other defects. 
The interfacial dislocation network plays a decisive role in the stationary creep properties of superalloys. Finely spaced dislocation networks can protect the $\gamma^{\prime}$ phase from being sheared by pairs of matrix dislocations $[15,10]$. However, if the dislocation network contains many long, straight dislocation segments, these can actually serve as partners for gliding matrix dislocations to penetrate the $\gamma^{\prime}$ phase [10, 66]. The interaction of $\mathrm{d}_{1}$ with the orthogonal misfit dislocation is a special case of such a process where the matrix dislocation and the misfit dislocation together cut into the $\gamma^{\prime}$ precipitate. In this case the dislocation penetrates the $\gamma^{\prime}$ phase on the initial glide plane of the matrix dislocation and on the cross-slip plane of the dissociated misfit dislocation. This is a clear example of how the core structure of interfacial dislocations can influence the interaction with channel dislocations. Due to the dependence of the misfit dislocation core structure on the Burgers vector sign, such a simultaneous cutting in on two glide planes could, however, only be expected for Co-base superalloys with positive lattice misfit.

When the dislocation $\mathrm{d}_{1}$ arrives at the interface, it intersects with the orthogonal misfit dislocations and gets pinned locally. Between the intersection nodes, $\mathrm{d}_{1}$ is, however, free to move and penetrates the $\gamma^{\prime}$ phase as leading superpartial dislocation $\mathrm{d}_{1}^{\prime}$. The dislocation can, additionally, relatively easily depin from the misfit dislocations, either by a reaction with $\mathrm{d}_{2}$, or by bowing out in a process similar to the Orowan mechanism (see Fig. 5, and Supplementary Movie M8 where this process is revealed in the 3D sample with planar IPB). Similar to the interaction of dislocations with forest dislocations which do not lead to the formation of strong locks [59], the intersection of the $60^{\circ}$ dislocation with the edge misfit dislocation does not act as a strong obstacle.

The situation is different where $d_{1}$ interacted with a misfit dislocation of the same Burgers vector in a collinear reaction. This reaction changed the topology of the dislocation arrangement, leading to a gap in the deposited matrix dislocation. The first superpartial dislocation $\mathrm{d}_{1}^{\prime}$ is effectively pinned by its connection to the orthogonal misfit dislocation. The second matrix dislocation $\mathrm{d}_{2}$ can unpin $\mathrm{d}_{1}^{\prime}$ in a second collinear interaction. As a result, however, $\mathrm{d}_{2}$ merges with $\mathrm{d}_{1}^{\prime}$, and $\mathrm{d}_{2}^{\prime}$ is connected to the misfit dislocation, i.e., to unpin $\mathrm{d}_{2}^{\prime}$ from the misfit dislocation, a third lattice dislocation with same Burgers vector and glide plane would be necessary. Similar to the situation in single crystals where collinear interactions were shown to significantly contribute to work hardening [60], collinear interactions with the misfit dislocation network seem to form strong obstacles to further cutting of the $\gamma^{\prime}$ phase, which can not be overcome by unzipping or Orowan bowing.

So far, the focus has been on a qualitative analysis of the mechanisms of dislocation-precipitate interaction. These mechanisms are governed by crystallography and are only a minimally influenced by the local chemical composition. The non-stoichiometric APT-informed atomistic sample shows, however, clear differences to the stoichiometric sample in terms of the strain at which the mechanisms take place. These quantitative differences are mainly caused by an increased Young's modulus due to the increased Al contents, and due to the decreased local misfit stresses in the non-stoichiometric sample. Furthermore, the chemical composition will affect the line tension of the dislocations due to its effect on the elastic constants, and the local chemical composition will also influence the APB energy and thereby the critical resolved stress required for matrix dislocations to cut into the precipitate. A detailed quantitative study of these effects will be presented elsewhere.

As with all atomistic simulations, the results of the MD studies can not be directly compared to experiments and need to be critically discussed. The usual size limitations of simulation boxes require large strains, and the typical atomistic time scales lead to strain rates which are many orders of magnitude higher than in experiments. Thermally activated processes can thus not be captured by MD simulations. Furthermore, the EAM potential might not exactly represent the interatomic bonding in all situations, and the Ni-Al-system does not adequately capture the chemical complexity of contemporary superalloys. However, the described processes are very robust, i.e., they are observed under significantly different simulation conditions, including different strain rates, temperatures and local chemical compositions. They can all, furthermore, be explained using arguments solely based on crystallography and dislocation theory. Consequently, we suggest that observations such as the role of the local IPB curvature for the misfit dislocation network and the importance of collinear interactions for protecting $\gamma^{\prime}$ precipitates can be generalized to the experimental situation.

Our study has implications not only for understanding the mechanical properties of superalloys, but also 
(a)

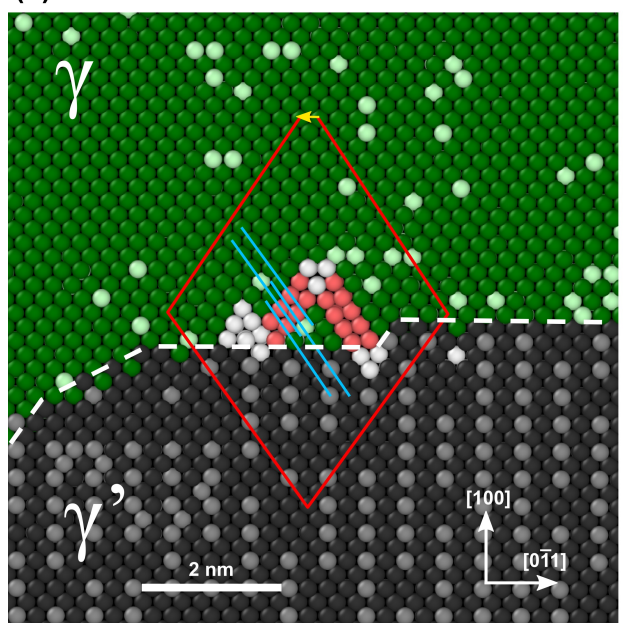

(b)

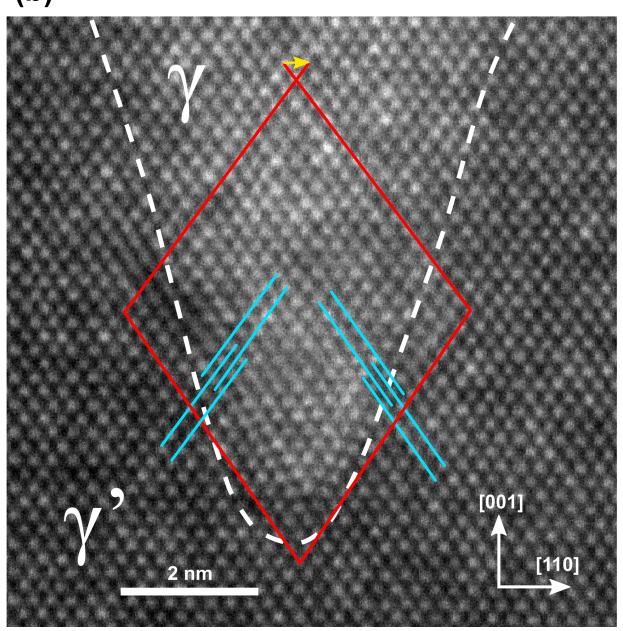

Figure 7: Lomer-Cottrell lock-like dislocation core structure in a Ni-base superalloy. (a) cut through the misfit dislocation in the APT-informed sample shown in Fig. 3(a). See Fig. 6 for the colour code. (b) STEM micrograph of a misfit dislocation at an interface groove in a LEK94 single crystal crept along $\langle 110\rangle$ at high-temperature. The Burgers circuit is indicated by the red line, the Burgers vector by the yellow arrow. The blue lines are added to highlight the stacking faults of the respective partial dislocations. Due to the negative lattice misfit in the LEK94 alloy, the Burgers vector of the misfit dislocation is opposite to the one in (a), leading to a LC lock-like core structure where the Shockley partial dislocations are oriented towards the $\gamma$ phase.

for modeling and simulation in general. By using different setups, the simulations clearly demonstrated that the usual, idealized sample geometries fail to reproduce key experimental observations such as the specific core structure of interfacial dislocations. Atom probe tomography informed samples for atomistic or mesoscale simulations help circumvent the need for overly simplified setups by providing a unique possibility to study dislocation processes directly in more realistic, complex microstructures.

\section{Conclusions}

We presented a novel approach to construct atom probe tomography informed atomistic simulation samples which avoids the lattice rectification step by using complementary information on the crystallographic structure of the specimen. Molecular dynamics simulations on real and idealized $\gamma / \gamma^{\prime}$ microstructures of a Ni-base superalloy showed that the local interface curvature and the local chemical composition critically influences the misfit dislocation network which forms on top of the $\gamma^{\prime}$ precipitate and the core structure of the misfit dislocations. In particular, misfit dislocations with Burgers vectors that are not an element of the $\{100\}$ interface plane and Lomer-Cottrell lock-like dislocation core structures could be observed, in agreement with TEM observations. The nature of the misfit dislocations in turn determines the interactions of matrix dislocations with the misfit dislocation network. Simulations of tensile tests at $1250 \mathrm{~K}$ with one or two $60^{\circ}$ matrix dislocations revealed the atomic scale details of many experimentally observed dislocation precipitate interaction mechanisms like the cutting of precipitates by a pair of superpartial dislocations, the pinning of threading dislocations and the knitting out of misfit dislocations, which cannot be reproduced by typically used, highly idealized simulation setups with planar interfaces. Notably, the simulations provided direct evidence for the importance of collinear dislocation interactions in protecting the $\gamma^{\prime}$ precipitates against dislocation cutting.

\section{Acknowledgments}

We thank J.J. Möller for the calculation of the concentration-dependent stacking fault energies and helpful discussions. The authors acknowledge support by the Deutsche Forschungsgemeinschaft (DFG) 
through projects C3 (atomistic simulations), A4 (APT) and A7 (HRTEM) of SFB/Transregio 103 (Single Crystal Superalloys).

\section{Appendix A. Supplementary data}

Supplementary data associated with this article can be found, in the online version.

\section{References}

[1] M. Durand-Charre, The Microstructure of Superalloys, CRC Press, 1998.

[2] R. C. Reed, The Superalloys: Fundamentals and Applications, Cambridge University Press, 2006.

[3] T. Murakumo, T. Kobayashi, Y. Koizumi, H. Harada, Acta Materalia 52 (2004) 3737-3744.

[4] C. Carry, J. Strudel, Acta Metallurgica 25 (1977) 767-777.

[5] M. Kolbe, A. Dlouhy, G. Eggeler, Materials Science and Engineering: A 246 (1998) 133-142.

[6] Z. Zhu, H. Basoalto, N. Warnken, R. Reed, Acta Materialia 60 (2012) 4888-4900.

[7] T. Gabb, S. Draper, D. Hull, Materials Science and Engineering A 118 (1989) 59-69.

[8] R. D. Field, T. M. Pollock, W. H. Murphy, The Development of $\gamma / \gamma$ ' Interfacial Dislocation Networks During Creep in Ni-Base Superalloys, in: Superalloys, 1992.

[9] C. Mayr, G. Eggeler, A. Dlouhy, Materials Science and Engineering: A 207 (1996) 51-63.

[10] L. Carroll, Q. Feng, T. Pollock, Metallurgical and Materials Transactions A 39 (2008) 1290-1307.

[11] F. Nabarro, Metallurgical and Materials transactions A 27 (1996) 513-530.

[12] R. Reed, D. Cox, C. Rae, Materials Science and Technology 23 (2007) 893-902.

[13] R. Srinivasan, G. F. Eggeler, M. J. Mills, Acta Materialia 48 (2000) 4867-4878.

[14] M. Kamaraj, Sadhana 28 (2003) 115-128.

[15] J. X. Zhang, T. Murakumo, Y. Koizumi, T. Kobayashi, H. Harada, S. Masaki, Metallurgical and Materials Transactions A 33 (2002) 3741-3746.

[16] K. Yashiro, M. Naito, Y. Tomita, International Journal of Mechanical Sciences 44 (2002) 1845-1860.

[17] T. Zhu, C.-y. Wang, Physical Review B 72 (2005) 014111.

[18] J. R. Djuansjah, K. Yashiro, Y. Tomita, Materials Transactions 49 (2008) 2507-2514.

[19] H. X. Xie, C. Y. Wang, T. Yu, Modelling and Simulation in Materials Science and Engineering 17 (2009).

[20] W. Wu, Y. Guo, Y. Wang, Philosophical Magazine 91 (2011) 357-372.

[21] J. P. Du, C. Y. Wang, T. Yu, Modelling and Simulation in Materials Science and Engineering 21 (2013) 015007.

[22] Y. Zhu, Z. Li, M. Huang, Computational Materials Science 70 (2013) 178-186.

[23] E. Bitzek, J. R. Kermode, P. Gumbsch, International Journal of Fracture (2015) Article in press.

[24] J. J. Möller, E. Bitzek, Engineering Fracture Mechanics Under Review (2015).

[25] A. Sedlmayr, E. Bitzek, D. S. Gianola, G. Richter, R. Mönig, O. Kraft, Acta Materialia 60 (2012) 3985-3993.

[26] T. Xu, M. Li, Philosophical Magazine 90 (2010) 2191-2222.

[27] T. F. Kelly, M. K. Miller, Review of scientific instruments 78 (2007) 031101.

[28] D. N. Seidman, Annual Review of Materials Research 37 (2007) 127-158.

[29] C. Pareige, F. Soisson, G. Martin, D. Blavette, Acta Materialia 47 (1999) 1889-1899.

[30] Z. Mao, C. K. Sudbrack, K. E. Yoon, G. Martin, D. N. Seidman, Nature materials 6 (2007) 210-6.

[31] X. Zhou, H. Wadley, R. Johnson, Acta Materialia 49 (2001) 4005-4015.

[32] W. Geng, D. Ping, Y. Gu, C. Cui, H. Harada, Physical Review B 76 (2007) 224102.

[33] Y. Amouyal, Z. Mao, C. Booth-Morrison, D. N. Seidman, Applied Physics Letters 94 (2009) 041917.

[34] B. Gault, X. Y. Cui, M. P. Moody, F. De Geuser, C. Sigli, S. P. Ringer, A. Deschamps, Scripta Materialia 66 (2012) 903-906.

[35] M. P. Moody, A. V. Ceguerra, A. J. Breen, X. Y. Cui, B. Gault, L. T. Stephenson, R. K. W. Marceau, R. C. Powles, S. P. Ringer, Nature communications 5 (2014) 5501.

[36] F. Vurpillot, L. Renaud, D. Blavette, Ultramicroscopy 95 (2003) 223-229.

[37] M. P. Moody, B. Gault, L. T. Stephenson, R. K. W. Marceau, R. C. Powles, A. V. Ceguerra, A. J. Breen, S. P. Ringer, Microscopy and Microanalysis 17 (2011) 226-239.

[38] A. V. Ceguerra, M. P. Moody, R. C. Powles, T. C. Petersen, R. K. W. Marceau, S. P. Ringer, Acta Crystallographica Section A 68 (2012) 547-560.

[39] A. B. Parsa, P. Wollgramm, H. Buck, C. Somsen, A. Kostka, I. Povstugar, P.-P. Choi, D. Raabe, A. Dlouhy, J. Müller, E. Spiecker, K. Demtroder, J. Schreuer, K. Neuking, G. Eggeler, Advanced Engineering Materials (2014) 1-15.

[40] D. J. Larson, T. J. Prosa, R. M. Ulfig, B. P. Geiser, T. F. Kelly, Local Electrode Atom Probe Tomography, Springer New York, New York, NY, 2013.

[41] M. Herbig, D. Raabe, Y. J. Li, P. Choi, S. Zaefferer, S. Goto, Physical Review Letters 112 (2014) 126103.

[42] E. V. Chernyaev, Marching Cubes 33: Construction of Topologically Correct Isosurfaces, Technical Report, CN/95-17, CERN, Institute for High Energy Physics, 1995.

[43] W. E. Lorensen, H. E. Cline, Computer Graphics 21 (1987) 163-169.

[44] D. Feng, L. Tierney, Journal of Statistical Software 28 (2008). 
[45] A. Prakash, M. Hummel, S. Schmauder, E. Bitzek, Nanosculpt: A tool to generate atomistic samples of arbitrary enclosed volumes, 2015. URL: http://www.gmp.ww.uni-erlangen.de/nanoSCULPT.php.

[46] A. Prakash, M. Hummel, S. Schmauder, E. Bitzek, Unpublished results (2015).

[47] J. O'Rourke, Computational Geometry in C, 2nd ed., Cambridge University Press, 1998.

[48] Y. Mishin, Acta Materialia 52 (2004) 1451-1467.

[49] P. M. Derlet, H. Van Swygenhoven, Physical Review B 67 (2003) 014202.

[50] E. Bitzek, P. Gumbsch, Mater. Sci. Eng. A 387-389 (2004) 11-15.

[51] E. Bitzek, P. Koskinen, F. Gähler, M. Moseler, P. Gumbsch, Physical Review Letters 97 (2006).

[52] W. Hoover, Physical Review A 31 (1985) 1695-1697.

[53] C. Begau, A. Hartmaier, E. George, G. Pharr, Acta Materialia 59 (2011) 934-942.

[54] J. Amodeo, C. Begau, E. Bitzek, Materials Research Letters 2 (2014) 140-145.

[55] A. Stukowski, Modelling and Simulation in Materials Science and Engineering 18 (2010) 015012.

[56] T. Link, a. Epishin, M. Klaus, U. Brückner, A. Reznicek, Materials Science and Engineering: A 405 (2005) $254-265$.

[57] A. Epishin, T. Link, G. Nolze, Journal of microscopy 228 (2007) 110-117.

[58] L. Agudo Jácome, P. Nörtershäuser, J.-K. Heyer, A. Lahni, J. Frenzel, A. Dlouhy, C. Somsen, G. Eggeler, Acta Materialia 61 (2013) 2926-2943.

[59] J. Hirth, J. Lothe, Theory of Dislocations, 2 ed., Krieger, Malabar, 1982.

[60] R. Madec, B. Devincre, L. Kubin, T. Hoc, D. Rodney, Science 301 (2003) 1879-1882.

[61] G. Mälzer, R. Hayes, T. Mack, G. Eggeler, Metallurgical and Materials Transactions A 38 (2007) 314-327.

[62] J. Sato, T. Omori, K. Oikawa, I. Ohnuma, Science 312 (2006) 90-91.

[63] A. Bauer, S. Neumeier, F. Pyczak, M. Göken, Scripta Materialia 63 (2010) 1197-1200.

[64] M. Huang, Z. Cheng, J. Xiong, J. Li, J. Hu, Z. Liu, J. Zhu, Acta Materialia 76 (2014) 294-305.

[65] R. G. Hoagland, T. E. Mitchell, J. P. Hirth, H. Kung, Philosophical Magazine A 82 (2002) 643-664.

[66] L. Agudo Jácome, P. Nörtershäuser, C. Somsen, A. Dlouhý, G. Eggeler, Acta Materialia 69 (2014) 246-264.

[67] K. Yashiro, F. Kurose, Y. Nakashima, K. Kubo, Y. Tomita, H. Zbib, International Journal of Plasticity 22 (2006) 713-723.

[68] K. Yashiro, M. Konishi, Y. Tomita, Computational Materials Science 43 (2008) 481-488.

[69] B. Liu, D. Raabe, F. Roters, A. Arsenlis, Acta Materialia 79 (2014) 216-233. 